\title{
Effect of Rotation for Two-Temperature Generalized Thermoelasticity of Two-Dimensional under Thermal Shock Problem
}

\author{
Kh. Lotfy ${ }^{1,2}$ and Wafaa Hassan ${ }^{2,3}$ \\ ${ }^{1}$ Department of Mathematics, Faculty of Science, P.O. Box 44519, Zagazig University, Zagazig, Egypt \\ ${ }^{2}$ Department of Mathematics, Faculty of Science and Arts, Al-mithnab, Qassim University, P.O. Box 931, \\ Al-mithnab Buridah 51931, Saudi Arabia \\ ${ }^{3}$ Mathematics and Physics Department, Faculty of Engineering, Port Said University, Port Said, Egypt \\ Correspondence should be addressed to Kh. Lotfy; khlotfy_1@yahoo.com
}

Received 6 July 2013; Accepted 3 September 2013

Academic Editor: Franklin Mendivil

Copyright (C) 2013 Kh. Lotfy and W. Hassan. This is an open access article distributed under the Creative Commons Attribution License, which permits unrestricted use, distribution, and reproduction in any medium, provided the original work is properly cited.

The theory of two-temperature generalized thermoelasticity based on the theory of Youssef is used to solve boundary value problems of two-dimensional half-space. The governing equations are solved using normal mode method under the purview of the LordŞhulman (LS) and the classical dynamical coupled theory (CD). The general solution obtained is applied to a specific problem of a half-space subjected to one type of heating, the thermal shock type. We study the influence of rotation on the total deformation of thermoelastic half-space and the interaction with each other under the influence of two temperature theory. The material is homogeneous isotropic elastic half-space. The methodology applied here is use of the normal mode analysis techniques that are used to solve the resulting nondimensional coupled field equations for the two theories. Numerical results for the displacement components, force stresses, and temperature distribution are presented graphically and discussed. The conductive temperature, the dynamical temperature, the stress, and the strain distributions are shown graphically with some comparisons.

\section{Introduction}

The linear theory of elasticity is of paramount importance in the stress analysis of steel, which is the commonest engineering structural material. To a lesser extent, linear elasticity describes the mechanical behavior of the other common solid materials, for example, concrete, wood, and coal. However, the theory does not apply to the behavior of many of the new synthetic materials of the clastomer and polymer type, for example, polymethyl-methacrylate (Perspex), polyethylene, and polyvinyl chloride. The linear theory of micropolar elasticity is adequate to represent the behavior of such materials. For ultrasonic waves, that is, for the case of elastic vibrations characterized by high frequencies and small wavelengths, the influence of the body microstructure becomes significant; this influence of microstructure results in the development of new type of waves that are not in the classical theory of elasticity. Metals, polymers, composites, solids, rocks, and concrete are typical media with microstructures. More generally, most of the natural and manmade materials including engineering, geological, and biological media possess a microstructure.

The classical coupled thermoelasticity theory proposed by Biot [1] with the introduction of the strain-rate term in the Fourier heat conduction equation leads to a parabolic-type heat conduction equation, called the diffusion equation. This theory predicts finite propagation speed for elastic waves but an infinite speed for thermal disturbance. This is physically unrealistic. To overcome such an absurdity, generalized thermoelasticity theories have been propounded by Lord and Shulman [2] as well as Green and Lindsay [3] advocating the existence of finite thermal wave speed in solids.

These theories have been developed by introducing one or two relaxation times in the thermoelastic process, either by modifying Fourier's heat conduction equation or by correcting the energy equation and Neuman-Duhamel relation. 
According to these generalized theories, heat propagation can be visualized as a wave phenomenon rather than a diffusion one; in the literature, it is usually referred to as the second sound effect. These two theories are structurally different from one another and one cannot be obtained as a particular case of the other. Various problems characterizing these theories have been investigated and has revealed some interesting phenomena. Brief reviews of this topic have been reported by Chandrasekharaiah and Srinath [4] and Chandrasekharaiah and Murthy [5]. The interplay of the Maxwell electromagnetic field with the motion of deformable solids is largely being undertaken by many investigators owing to the possibility of its application to geophysical problems and certain topics in optics and acoustics. Moreover, the earth is subject to its own magnetic field and the material of the earth may be electrically conducting. Thus, the magnetoelastic nature of the earth's material may affect the propagation of waves. Many authors have considered the propagation of electromagnetothermoelastic waves in an electrically and thermally conducting solid. A comprehensive review of the earlier contributions to the subject can be found in the study by Puri [6]. Among the authors who considered the generalized magnetothermoelastic equations are Nayfeh and Nemat-Nasser [7] who studied the propagation of plane waves in a solid under the influence of an electromagnetic field. They have obtained the governing equations in the general case and the solution for some particular cases. Roy Choudhuri and Mukhopdhyay [8] extended these results to rotating media. Ezzat [9] has studied the problem of generation of generalized magnetothermoelastic waves by thermal shock in a perfectly conducting half-space. Ezzat et al. [10] have established the model of two dimensional equations of generalized magnetothermoelasticity. In dealing with classical or generalized thermoelastic problems in most situations, the displacement potential function approach is used. However Bahar and Hetnarski [11, 12] outlined several disadvantages of the potential function approach. These may be summarized in the fact that the boundary and initial conditions of the problem are not related directly to the potential function, as it has no physical meaning explicitly.

Secondly, more stringent assumptions must be made on the behaviour of potential functions than on the actual physical quantities. Last of all, it was found that many integral representations of physical quantities are convergent in the classical sense while their potential function representations only converge in the mean. To get rid of these difficulties, Bahar and Hetnarski [13] introduced the state space formulation in thermoelastic problems. This state space approach has been further developed in Sherief [14] to include the effect of heat sources. Sherief and Anwar [15] surveyed a twodimensional thermal shock problem for a semi-infinite piezoelectric rod using state space approach. Youssef and El-bary [16] put forward an analysis for a generalized thermoelastic infinite layer problem under three theories using state space approach. State space formulation to the vibration of gold nanobeam in femtoseconds scale was done by Elsibai and Youssef [17].

The theory of heat conduction in a deformable body, formulated by Chen and Gurtin [18] and Chen et al. [19, 20] depends on two different temperatures, the conductive temperature and the thermo dynamical temperature. Chen et al. [21] have suggested that the difference between these two temperatures is proportional to heat supply. In absence of heat supply, these two temperatures are identical for time independent situation. However, for time dependent cases, particularly for problems related to wave propagation, the two temperatures, are in general, different, regardless of heat supply. The two temperature thermoelasticity theory has gained much attention of the researchers in the recent years. The existence, structural stability, convergence, and spatial behaviour of two temperature thermoelasticity have been provided by Quintanilla and Tien [22]. Youssef [23] has developed a new model of generalized thermoelasticity that depends on two temperatures $T$ and $\phi$, where the difference between the two temperatures is proportional to heat supply $\ddot{\phi}_{, i i}$ with a nonnegative constant $a$ (length $^{2}$ ).

Later on, Youssef and Al-Lehaibi [24], Misra et al. [25], Singh [26], and Singh and Bala [27] investigated various problems on the basis of two temperature thermoelasticity with relaxation times and showed that the obtained results are qualitatively different as compared to those in case of one temperature thermoelasticity.

Roy Choudhuri and Debnath [28, 29], Othman [30, 31] Othman and Singh [32], and Othman and Song [33] studied the effect of rotation in a micropolar-generalized thermoelastic and thermoviscoelasticity half-space under different theories. The propagation of plane harmonic waves in a rotating elastic medium without thermal field has been studied. It was shown there that the rotation causes the elastic medium to be dispersive and an isotropic. These problems are based on more realistic elastic model since earth, moon, and other plants have angular velocity.

Owing to the mathematical difficulties encountered in two- and three-dimensional multifield coupled generalized heat conduction problems, the problems become too complicated to obtain an analytical solution. Instead of analytical methods, several authors have applied numerical methods such as finite difference method, finite element method and boundary value method. For solving such kind of problems, one can find several two-dimensional works based on the generalized thermoelasticity by using the normal mode analysis in the literature Ezzat and Abd Elall [34], Othman et al. [35, 36], Lotfy and Othman [37], Lotfy et al. [38-40], and Sarkar and Lahiri [41]. Using the normal mode analysis technique, we will get the solution in the Fourier transformed domain actually. To apply the normal mode analysis, we have to assume that all the relations are sufficiently smooth on the real axis such that the normal mode analysis of all these functions exists. The normal mode analysis [34-40] was used to obtain the exact expression for the temperature distribution, thermal stresses, and the displacement components.

The present paper is intended to demonstrate the use of the normal mode analysis in analyzing the propagation of thermoelastic waves in two temperature theory of thermoelasticity under the effect of rotation. Normal mode analysis is employed to obtain the exact solution. Numerical values of the field quantities are calculated by considering illustrative 
examples and their variations with respect to space coordinate are displayed graphically and discussed under thermal shock problem.

\section{Formulation of the Problem}

In the present paper, the authors consider the problem of a homogeneous, isotropic, elastic half-space $(x \geq 0)$. The surface of the half-space is subjected initially $(t=0)$ to a thermal shock that is a function of $y$ and $t$. Thus all the quantities considered in this problem will be functions of the time variable $t$ and coordinates $x, y$. We can introduce the equations of the problem as follows.

The heat conduction equation takes the form Youssef [23]:

$$
K \varphi_{, i i}=\left(\frac{\partial}{\partial t}+\tau_{0} \frac{\partial^{2}}{\partial t^{2}}\right)\left(\rho C_{E} T+\gamma T_{0} u_{i, j}\right) .
$$

The constitutive equation takes the form

$$
\sigma_{i j}=\lambda e_{k k} \delta_{i j}+2 \mu e_{i j}-\gamma T \delta_{i j}
$$

Since the medium is rotating uniformly with an angular velocity $\underline{\Omega}=\Omega \underline{n}$, where $\underline{n}$ is a unit vector representing the direction of the axis of rotation. The displacement equation of motion in the rotating frame of reference has two additional terms, centripetal acceleration, $\underline{\Omega} \times(\underline{\Omega} \times \underline{u})$ due to timevarying motion only and the Coriolis acceleration $2 \underline{\Omega} \times \underline{\dot{u}}$, where $\underline{u}$ is the dynamic displacement vector.

The equations of motion in a rotating frame of reference in the context of generalized thermo elasticity are

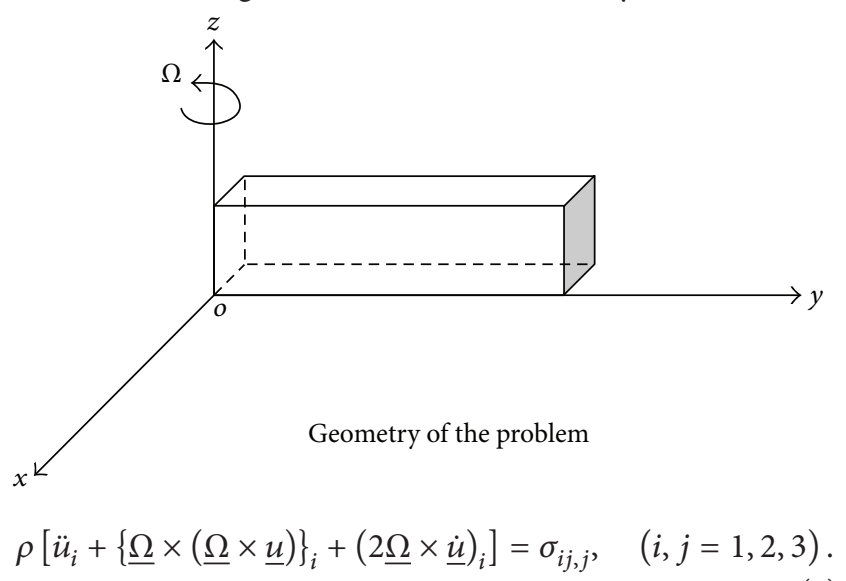

The relation between the heat conduction and the dynamical heat takes the form

$$
\varphi-T=a \varphi_{, i i},
$$

where $a>0$ two-temperature parameter, Youssef [23].

Now, we will suppose elastic and homogenous half-space $x \geq 0$ which obey (1)-(4) and initially quiescent where all the state functions are depend only, on the dimension, $x, y$ and the time $t$.

The displacement components for one dimension medium have the form

$$
u_{x}=u(x, y, t), \quad u_{y}=v(x, y, t), \quad u_{z}=0 .
$$

The strain component takes the form

$$
e_{i j}=\frac{1}{2}\left(u_{i, j}+u_{j, i}\right)
$$

The heat conduction equation takes the form

$$
\begin{aligned}
& K\left(\frac{\partial^{2} \varphi}{\partial x^{2}}+\frac{\partial^{2} \varphi}{\partial y^{2}}\right) \\
& =\left(\frac{\partial}{\partial t}+\tau_{0} \frac{\partial^{2}}{\partial t^{2}}\right) \rho C_{E} T \\
& \quad+\gamma T_{0}\left(\frac{\partial}{\partial t}+\tau_{0} \frac{\partial^{2}}{\partial t^{2}}\right)\left(\frac{\partial u}{\partial x}+\frac{\partial v}{\partial y}\right) .
\end{aligned}
$$

The constitutive law equations can be written as

$$
\begin{gathered}
\sigma_{x x}=(2 \mu+\lambda) \frac{\partial u}{\partial x}+\lambda \frac{\partial v}{\partial y}-\gamma T \\
\sigma_{y y}=(2 \mu+\lambda) \frac{\partial v}{\partial y}+\lambda \frac{\partial u}{\partial x}-\gamma T \\
\sigma_{x y}=\mu\left(\frac{\partial u}{\partial y}+\frac{\partial v}{\partial x}\right) .
\end{gathered}
$$

Using the summation convection. From (8), we note that the third equation of motion in (3) is identically satisfied and first two equations become

$$
\begin{aligned}
& \rho\left(\frac{\partial^{2} u}{\partial t^{2}}-\Omega^{2} u+2 \Omega \dot{v}\right)=\mu \nabla^{2} u+(\mu+\lambda) \frac{\partial e}{\partial x}-\gamma \frac{\partial T}{\partial x} \\
& \rho\left(\frac{\partial^{2} v}{\partial t^{2}}-\Omega^{2} v-2 \Omega \dot{u}\right)=\mu \nabla^{2} v+(\mu+\lambda) \frac{\partial e}{\partial y}-\gamma \frac{\partial T}{\partial y}
\end{aligned}
$$

The relation between the heat conduction and dynamical heat takes the form

$$
\varphi-T=a\left(\frac{\partial^{2} \varphi}{\partial x^{2}}+\frac{\partial^{2} \varphi}{\partial y^{2}}\right)
$$

To transform the above equations into nondimensional forms, we define the following non-dimensional variables:

$$
\begin{gathered}
\left(x^{\prime}, y^{\prime}, u^{\prime}, v^{\prime}\right)=c_{0} \eta(x, y, u, v), \\
\left(t^{\prime}, \tau_{0}^{\prime}, v_{0}^{\prime}\right)=c_{0}^{2} \eta\left(t, \tau_{0}, v_{0}\right), \\
\left(\theta^{\prime}, \varphi^{\prime}\right)=\frac{(T, \varphi)-T_{0}}{T_{0}}, \\
\sigma_{i j}^{\prime}=\frac{\sigma_{i j}}{2 \mu+\lambda}, \quad \Omega^{\prime}=\frac{\Omega}{c_{0}^{2} \eta},
\end{gathered}
$$

where $\eta=\left(\rho C_{E} / K\right), C_{2}^{2}=(\mu / \rho)$ and $C_{0}^{2}=(2 \mu+\lambda) / \rho$. 
Hence, we have (dropping the dashed for convenience)

$$
\begin{gathered}
\nabla^{2} \varphi-\left(1+\tau_{0} \frac{\partial}{\partial t}\right) \frac{\partial \theta}{\partial t}-\varepsilon\left(1+\tau_{0} \frac{\partial}{\partial t}\right) \frac{\partial e}{\partial t}=0 \\
\varphi-\theta=\beta\left(\frac{\partial^{2} \varphi}{\partial x^{2}}+\frac{\partial^{2} \varphi}{\partial y^{2}}\right)
\end{gathered}
$$

where $\varepsilon=\left(\gamma / \rho C_{E}\right)$ and $\beta=a \eta^{2} c_{0}^{2}$.

Assume the scalar potential functions $\Pi(x, y, t)$ and $\psi(x, y, t)$ defined by the relations in the nondimensional form

$$
u=\frac{\partial \Pi}{\partial x}+\frac{\partial \psi}{\partial y}, \quad v=\frac{\partial \Pi}{\partial y}-\frac{\partial \psi}{\partial x} .
$$

By using (15) and (12) in (9), we obtain

$$
\begin{aligned}
& {\left[\nabla^{2}+\Omega^{2}-\frac{\partial^{2}}{\partial t^{2}}\right] \Pi+2 \Omega \frac{\partial \psi}{\partial t}-a_{0} \theta=0,} \\
& \left(\nabla^{2}-a_{1} \frac{\partial^{2}}{\partial t^{2}}+a_{1} \Omega^{2}\right) \psi-2 \Omega a_{1} \frac{\partial \Pi}{\partial t}=0,
\end{aligned}
$$

where

$$
a_{1}=\frac{\rho C_{0}^{2}}{\mu}, \quad a_{0}=\frac{\gamma T_{0}}{\rho C_{0}^{2}} .
$$

The heat conduction equation (13) becomes

$$
\nabla^{2} \varphi-\left(1+\tau_{0} \frac{\partial}{\partial t}\right) \frac{\partial \theta}{\partial t}-\varepsilon\left(1+\tau_{0} \frac{\partial}{\partial t}\right) \frac{\partial \Pi}{\partial t}=0 .
$$

\section{Normal Mode Analysis}

The solution of the physical variable can be decomposed in terms of normal modes as the following way:

$$
\begin{aligned}
{\left[\Pi, \psi, \varphi, \theta, \sigma_{i j}\right](x, y, t) } \\
=\left[\Pi^{*}, \psi^{*}, u^{*}(x), \varphi^{*}(x), \theta^{*}(x), \sigma_{i j}^{*}(x)\right] \\
\quad \times \exp (\omega t+i b y),
\end{aligned}
$$

where $\omega$ is the (complex) time constant, $i$ is the imaginary, $b$ be a wave number in the $y$-direction and $\Pi^{*}, \psi^{*}, u^{*}(x)$, $\varphi^{*}(x), \theta^{*}(x)$, and $\sigma_{i j}^{*}(x)$ are the amplitude of the functions. By using the normal mode defined in (20), (17)-(19) and (14) take the following forms:

$$
\begin{gathered}
{\left[D^{2}-A_{1}\right] \Pi^{*}+A_{0} \psi^{*}-A_{2} \theta^{*}=0,} \\
\left(D^{2}-A_{4}\right) \psi^{*}-A_{5} \Pi=0 \\
{\left[D^{2}-A_{3}\right] \varphi^{*}=-\beta^{*} \theta^{*}} \\
\left(D^{2}-b^{2}\right) \varphi-A \theta^{*}-B \Pi^{*}=0
\end{gathered}
$$

where $A_{0}=2 \Omega \omega, A=\omega\left(1+\omega \tau_{0}\right), B=\varepsilon A, A_{1}=b^{2}+\omega^{2}-\Omega^{2}$, $A_{2}=a_{0}, A_{3}=\left(\beta b^{2}+1\right) / \beta, \beta^{*}=1 / \beta, A_{4}=b^{2}+a_{1}\left(\omega^{2}-\Omega^{2}\right)$, $A_{5}=a_{1} A_{0}$, and $D=d / d x$.
Eliminating $\theta^{*}(x), \Pi^{*}(x), \psi^{*}(x)$, and $\varphi^{*}(x)$ between (21) and (24), we obtain the partial differential equation satisfied by $\theta^{*}(x)$

$$
\left[D^{6}-E D^{4}+F D^{2}-G\right] \Pi^{*}(x)=0,
$$

where $A_{6}=\left(\beta^{*} b^{2}+A_{3} A\right) /\left(\beta^{*}+A\right), A_{7}=-B /\left(\beta^{*}+A\right)$.

Since

$$
\begin{gathered}
E=A_{1}+A_{4}+A_{6}+A_{2} A_{7}, \\
F=A_{1} A_{4}+A_{0} A_{5}+A_{6}\left(A_{1}+A_{4}\right)+A_{2} A_{7}\left(A_{3}+A_{4}\right), \\
G=A_{1} A_{4} A_{6}+A_{2} A_{3} A_{4} A_{7}+A_{0} A_{5} A_{6} .
\end{gathered}
$$

In a similar manner, we get

$$
\left[D^{6}-E D^{4}+F D^{2}-G\right]\left(\theta^{*}, \varphi^{*}, \psi^{*}\right)(x)=0 .
$$

The above equation can be factorized

$$
\left(D^{2}-k_{1}^{2}\right)\left(D^{2}-k_{2}^{2}\right)\left(D^{2}-k_{3}^{2}\right) \Pi^{*}(x)=0,
$$

where $k_{n}^{2}(n=1,2,3)$ are the roots of the following characteristic equation:

$$
k^{6}-E k^{4}+F k^{2}-G=0 .
$$

The solution of (28) which is bounded as $x \rightarrow \infty$ is given by

$$
\Pi^{*}(x)=\sum_{n=1}^{3} M_{n}(b, \omega) \exp \left(-k_{n} x\right)
$$

Similarly

$$
\begin{aligned}
& \theta^{*}(x)=\sum_{n=1}^{3} M_{n}^{\prime}(b, \omega) \exp \left(-k_{n} x\right), \\
& \psi^{*}(x)=\sum_{n=1}^{3} M_{n}^{\prime \prime}(b, \omega) \exp \left(-k_{n} x\right), \\
& \varphi^{*}(x)=\sum_{n=1}^{3} M_{n}^{\prime \prime \prime}(b, \omega) \exp \left(-k_{n} x\right) .
\end{aligned}
$$

Since,

$$
\begin{aligned}
& u^{*}(x)=D \Pi^{*}+i b \psi^{*}, \\
& v^{*}(x)=i b \Pi^{*}-D \psi^{*}, \\
& e^{*}(x)=D u^{*}+i b v^{*}
\end{aligned}
$$


Using (34) and (35), in order to obtain the amplitude of the displacement components $u$ and $v$, which are bounded as $x \rightarrow \infty$, then (34) and (35) become

$$
\begin{aligned}
u^{*}(x)= & -\sum_{n=1}^{3} M_{n}(b, \omega) k_{n} e^{-k_{n} x} \\
& +i b \sum_{n=1}^{3} M_{n}^{\prime \prime}(b, \omega) \exp \left(-k_{n} x\right), \\
v^{*}(x)= & i b \sum_{n=1}^{3} M_{n}(b, \omega) e^{-k_{n} x} \\
& +\sum_{n=1}^{3} M_{n}^{\prime \prime}(b, \omega) k_{n} e^{-k_{n} x}
\end{aligned}
$$

where $M_{n}, M_{n}^{\prime}, M_{n}^{\prime \prime}$, and $M_{n}^{\prime \prime \prime}$ are some parameters depending on $\beta, b$, and $\omega$.

Substituting from (30)-(32) into (21)-(24), we have

$$
\begin{array}{ll}
M_{n}^{\prime}(b, \omega)=H_{1 n} M_{n}(b, \omega), & n=1,2,3, \\
M_{n}^{\prime \prime}(b, \omega)=H_{2 n} M_{n}(b, \omega), & n=1,2,3, \\
M_{n}^{\prime \prime \prime}(b, \omega)=H_{3 n} M_{n}(b, \omega), & n=1,2,3,
\end{array}
$$

where

$$
\begin{gathered}
H_{1 n}=\frac{A_{7}\left(k_{n}^{2}-A_{3}\right)}{k_{n}^{2}-A_{6}}, \quad n=1,2,3, \\
H_{2 n}=\frac{A_{5}}{\left(k_{n}^{2}-A_{4}\right)}, \quad n=1,2,3, \\
H_{3 n}=\frac{A_{7} \beta^{*}}{A_{6}-k_{n}^{2}}, \quad n=1,2,3 .
\end{gathered}
$$

Thus, we have

$$
\begin{gathered}
\theta^{*}(x)=\sum_{n=1}^{3} H_{1 n} M_{n}\left(b, \beta^{*}, \omega\right) \exp \left(-k_{n} x\right), \\
\psi^{*}(x)=\sum_{n=1}^{3} H_{2 n} M_{n}\left(b, \beta^{*}, \omega\right) \exp \left(-k_{n} x\right), \\
\varphi^{*}(x)=\sum_{n=1}^{3} H_{3 n} M_{n}\left(b, \beta^{*}, \omega\right) \exp \left(-k_{n} x\right) .
\end{gathered}
$$

The stress components can be calculated by using (31) and (37) in (8) as follows

$$
\begin{aligned}
& \sigma_{x x}^{*}=\sum_{n=1}^{3} h_{n} M_{n}(b, \omega) \exp \left(-k_{n} x\right), \\
& \sigma_{y y}^{*}=\sum_{n=1}^{3} h_{n}^{\prime} M_{n}(b, \omega) \exp \left(-k_{n} x\right), \\
& \sigma_{x y}^{*}=\sum_{n=1}^{3} h_{n}^{\prime \prime} M_{n}(b, \omega) \exp \left(-k_{n} x\right) .
\end{aligned}
$$

The displacements components can be reduced by using (39) in (37) and we get

$$
\begin{aligned}
& u^{*}(x)=\sum_{n=1}^{3}\left(i b H_{2 n}-k_{n}\right) M_{n}(b, \omega) e^{-k_{n} x}, \\
& v^{*}(x)=\sum_{n=1}^{3}\left(i b+k_{n} H_{2 n}\right) M_{n}(b, \omega) e^{-k_{n} x},
\end{aligned}
$$

where

$$
\begin{gathered}
h_{n}=-\left[k_{n}\left(i b H_{2 n}-k_{n}\right)-\frac{i b \lambda\left(i b+k_{n} H_{2 n}\right)}{2 \mu+\lambda}+\frac{\gamma T_{0} H_{1 n}}{2 \mu+\lambda}\right], \\
h_{n}^{\prime}=\left[i b\left(k_{n} H_{2 n}+i b\right)-\frac{\lambda k_{n}\left(i b H_{2 n}-k_{n}\right)}{2 \mu+\lambda}-\frac{\gamma T_{0} H_{1 n}}{2 \mu+\lambda}\right], \\
h_{n}^{\prime \prime}=\frac{\mu\left[i b\left(i b H_{2 n}-k_{n}\right)-k_{n}\left(i b+k_{n} H_{2 n}\right)\right]}{2 \mu+\lambda} .
\end{gathered}
$$

The normal mode analysis is, in fact, to look for the solution in Fourier transformed domain. Assuming that all the field quantities are sufficiently smooth on the real line such that normal mode analysis of these functions exists.

\section{Application}

4.1. Thermal Shock Problem. In order to determine the constants $M_{n}(n=1,2,3)$. In the physical problem, we should suppress the positive exponentials that are unbounded at infinity. The constants $M_{1}, M_{2}$, and $M_{3}$ have to be chosen such that the boundary conditions on the surface at $x=0$ take the form

(1) thermal boundary conditions that the surface of the half-space subjected to thermal shock

$$
\theta(0, y, t)=f(0, y, t)
$$

(2) mechanical boundary condition that surface of the half-space is traction-free

$$
\sigma_{x x}(0, y, t)=0
$$

(3) mechanical boundary condition that surface of the half-space is traction-free

$$
\sigma_{x y}(0, y, t)=0
$$

where $f(0, y, t)$ is some given function in $y$ and $t$.

Substituting from the expressions of the considered variables into the above boundary conditions (48)-(50), we 
obtain the following equations satisfyed by the parameters after some simple manipulations

$$
\begin{gathered}
\sum_{n=1}^{3} H_{1 n} M_{n}\left(b, \beta^{*}, \omega\right)=f^{*}(y, t), \\
\sum_{n=1}^{3} h_{n} M_{n}(b, \beta, \omega)=0, \\
\sum_{n=1}^{3} h_{n}^{\prime \prime} M_{n}(b, \beta, \omega)=0 .
\end{gathered}
$$

Invoking the boundary conditions (51) at the surface $x=0$ of the plate, we obtain a system of three equations. After applying the inverse of matrix method, we have the values of the three constants $M_{j}, j=1,2,3$. Hence, we obtain the expressions of displacements, temperature distribution, and another physical quantity of the plate.

\section{Numerical Results}

In order to analyze the above problem numerically, we now consider a numerical example for which computational results are given. The results depict the variation of temperature, displacement, and stress fields in the context of two theories. To study the effect of rotation and two temperature on wave propagation. The copper material was chosen for the purpose of numerical example. The numerical constants (in SI unit) of the problem were taken as

$$
\begin{gathered}
\lambda=7.59 \times 10^{9} \mathrm{~N} / \mathrm{m}^{2}, \quad \mu=3.86 \times 10^{10} \mathrm{~kg} / \mathrm{ms}^{2}, \\
\rho=8954 \mathrm{~kg} / \mathrm{m}^{3}, \quad \tau_{0}=0.02 \mathrm{~s}, \\
\alpha=-1.28 \times 10^{9} \mathrm{~N} / \mathrm{m}^{2}, \quad \beta=0.32 \times 10^{9} \mathrm{~N} / \mathrm{m}^{2}, \\
\eta=8886.73 \mathrm{~m} / \mathrm{s}^{2}, \quad \varepsilon=0.0168, \\
\alpha_{t}=1.78 \times 10^{-5} \mathrm{~K}^{-1}, \quad k=386 \mathrm{Wm}^{-1} \mathrm{~K}^{-1}, \\
b=1, \quad C_{E}=383.1 \mathrm{~J} /(\mathrm{kgK}) \\
T_{0}=293 \mathrm{~K}, \quad f^{*}=1, \quad \omega=\omega_{0}+i \xi, \\
\omega_{0}=2, \quad \xi=1 .
\end{gathered}
$$

Since we have $\omega=\omega_{0}+i \xi$, where $i$ is the imaginary unit, $e^{\omega t}=e^{\omega_{0} t}(\cos \xi t+i \sin \xi t)$ and for small value of time, we can take $\omega=\omega_{0}$ (real).

The computations were carried out for $a$ value of time $t=0.1$. The numerical technique, outlined above, was used for the distribution of the real part of the thermal temperature $\theta$ and $\phi$, the displacement $u, v$, strain, and the stress $\left(\sigma_{x x}, \sigma_{y y}, \sigma_{x y}\right)$ distribution for the problem. The field quantities, temperature, displacement components, and stress components depend not only on space $x$ and time $t$ but also on the thermal relaxation time $\tau_{0}$. Here all the variables are taken in nondimensional forms.
In the first group Figures 1(a), 1(b), 1(c), 1(d), 1(e), 1(f), $1(\mathrm{~g})$, and $1(\mathrm{~h})$, the graph shows the two curves predicted by different theories of thermoelasticity. In these figures, the solid lines represent the solution in the Coupled theory, the dashed lines represent the solution in the generalized Lord and Shulman theory. We notice that the results for the temperature, the displacement, and stresses distribution when the relaxation time is including in the heat equation are distinctly different from those when the relaxation time is not mentioned in heat equation, because the thermal waves in the Fourier theory of heat equation travel with an infinite speed of propagation as opposed to finite speed in the non-Fourier case. This demonstrates clearly the difference between the coupled and the theory of thermoelasticity (LS).

The second group Figures 2(a), 2(b), 2(c), 2(d), 2(e), 2(f), $2(\mathrm{~g})$, and $2(\mathrm{~h})$ show the comparison between the thermal temperature $\theta$ and $\phi$, displacement components $u, v$, the force stresses components $\sigma_{x x}, \sigma_{y y}$, and $\sigma_{x y}$, the case of different two values of rotation, and constant of two temperature parameter $(\beta=1)$ under LS theory. It should be noted (Figure 2(a)) in this problem. It is clear from the graph that $\theta$ sharp decreases to minimum value at the beginning, where it experiences smooth increases (with maximum positive gradient). Graph lines for both values of rotation show different slopes. In other words, the temperature lines for $\Omega=0.0$ has the highest gradient when compared with that of $\Omega=0.2$ in all ranges. In addition, all lines begin to coincide when the horizontal distance $x$ increases to reach the reference temperature of the solid. These results obey physical reality for the behaviour of copper as a polycrystalline solid. Figure 2(b), the horizontal displacement $u$, despite the peaks (for different values of rotation), the magnitude of the maximum displacement peak strongly depends on the rotation. It is also clear that the rate of change of $u$ decreases with increasing the rotation. On the other hand, Figure 2(c) shows atonable increase of the vertical displacement $v$, near the beginning reachs minimum value and then reaching zero value at the infinity (state of particles equilibrium) when $\Omega=0.0$. Figure 2(d) displays a comparison of the strain in two cases which show the different behaviours: when $\Omega=$ 0.0 and $\Omega=0.2$ we can say that significant difference in the strain is noticed for different values of the rotation. In addition, all lines begin to coincide when the horizontal distance $x$ increases to reach zero at infinity. In Figure 2(e) the horizontal stresses $\sigma_{x x}$ graph lines for both values of rotation show different slopes. In other words, the $\sigma_{x x}$ component line for $\Omega=0.0$ has the highest gradient when compared with that of $\Omega=0.2$. In addition, all lines begin to coincide when the horizontal distance $x$ is increased to reach zero after their relaxations at infinity. Variation of $\Omega$ has a serious effect on both magnitudes of mechanical stresses. These trends obey elastic and thermoelastic properties of the solid under investigation. Figure 2(f) shows that the stress component $\sigma_{y y}$ takes the different behavior. In other words, the $\sigma_{y y}$ component line for $\Omega=0.0$ has the highest gradient when compared with that of $\Omega=0.2$. Figure $2(\mathrm{~g})$ shows that the stress component $\sigma_{x y}$ satisfies the boundary condition; it sharp decreases in the start and start increases (minimum) in the context of the $\Omega=0.2$ but when $\Omega=0.0$ take the different 


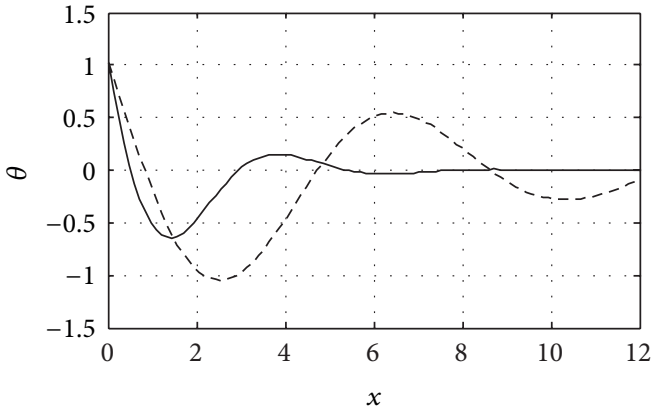

(a) The thermodynamical heat distribution

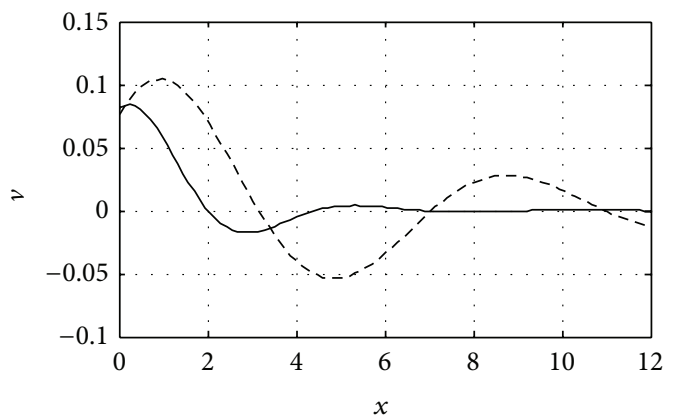

(c) The displacement distribution ( $v)$

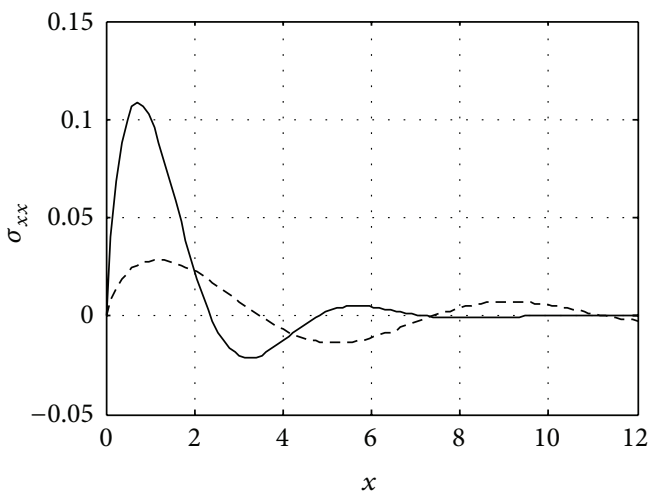

(e) The stress $\sigma_{x x}$ distribution

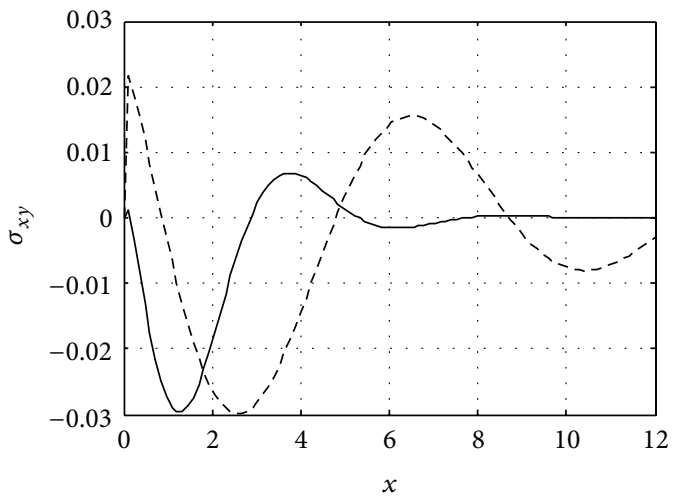

$$
\begin{aligned}
& - \text { CD } \\
& --- \text { LS }
\end{aligned}
$$

(g) The stress $\sigma_{x y}$ distribution

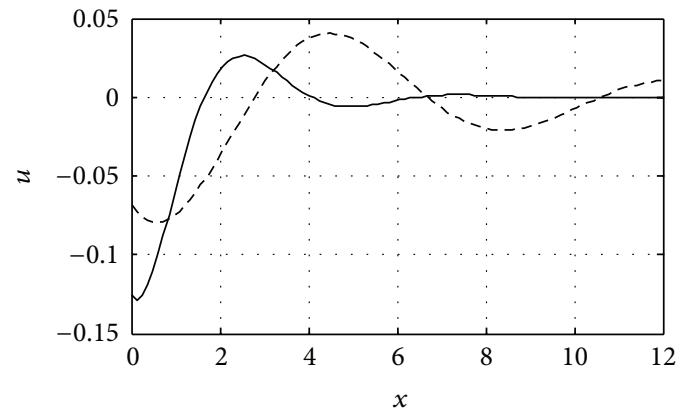

(b) The displacement distribution $(u)$

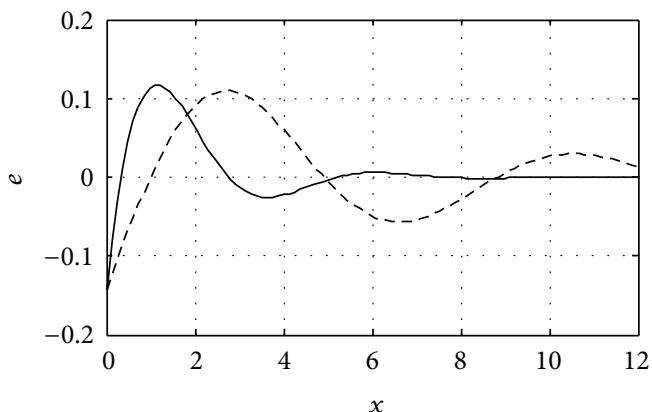

(d) The strain distribution (e)

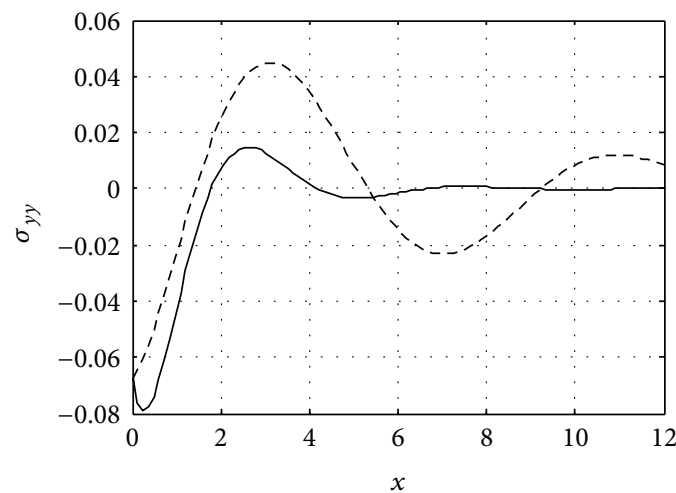

(f) The stress $\sigma_{y y}$ distribution

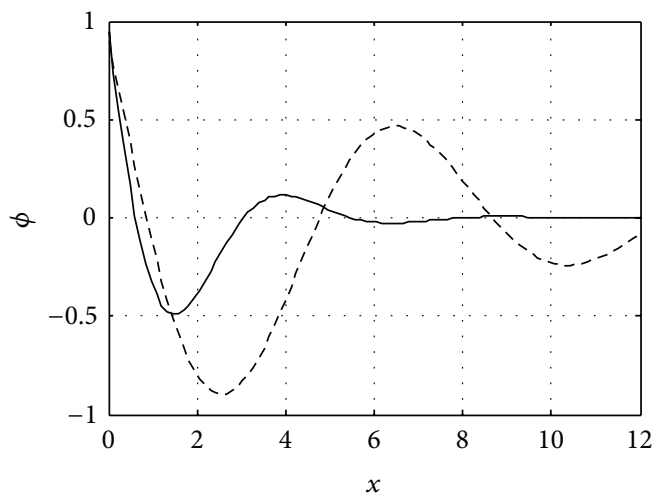

$\begin{array}{ll}- & \text { CD } \\ --- & \text { LS }\end{array}$

(h) The conductive heat distribution

FiguRE 1: The thermal temperature $\theta$, the displacement $u, v$ the strain $e$ distribution, the stresses distribution $\sigma_{y y}, \sigma_{x x}$, and $\sigma_{x y}$ and the thermal temperature $\phi$ distribution with $\beta=0.1$, and $t=0.1$ under CD and LS theories. 


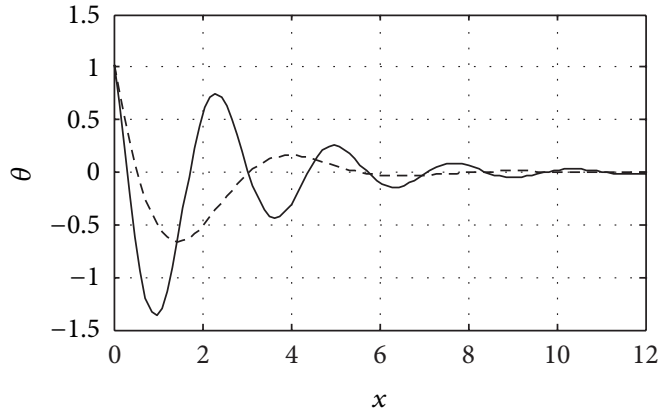

(a) The thermodynamical heat distribution

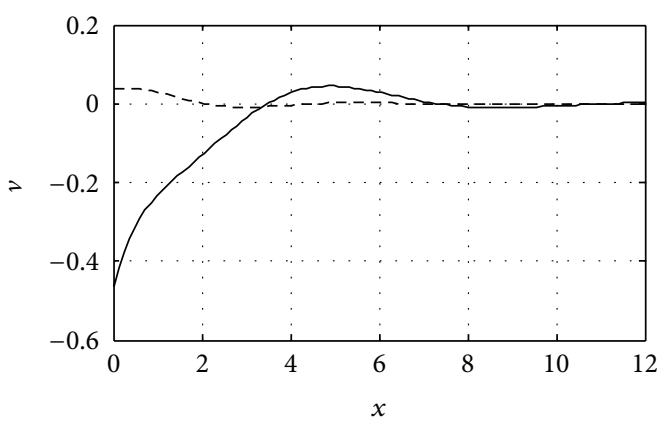

(c) The displacement distribution $(v)$

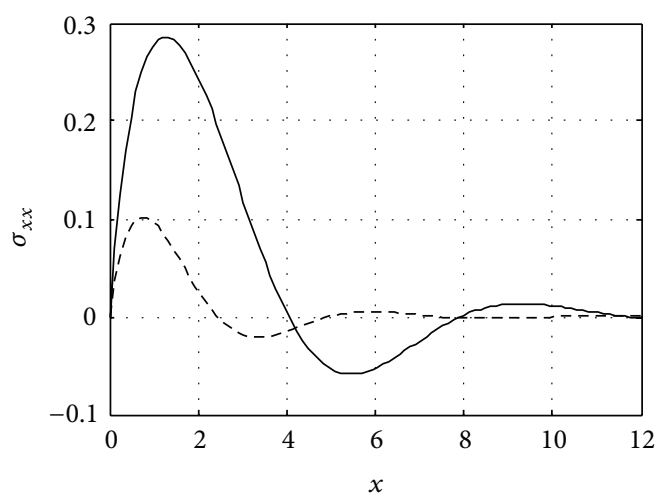

(e) The stress $\sigma_{x x}$ distribution

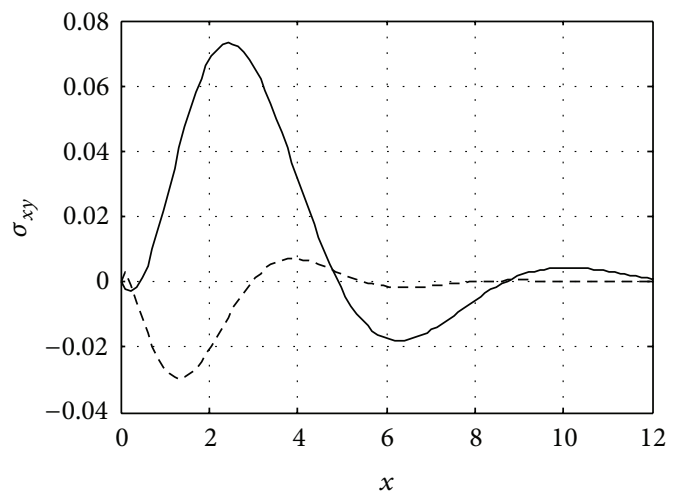

$\Omega=0.0$

$---\Omega=0.2$

(g) The stress $\sigma_{x y}$ distribution

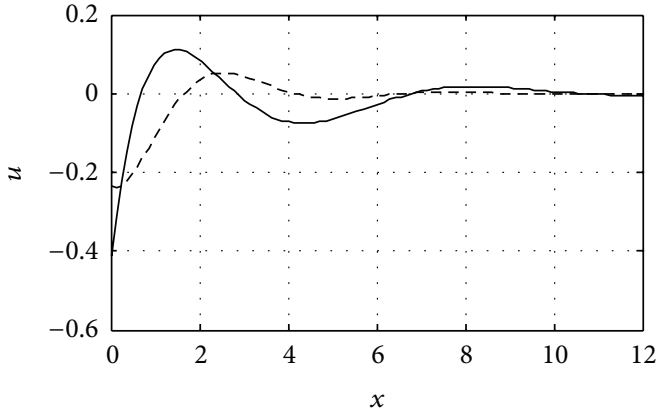

(b) The displacement distribution $(u)$

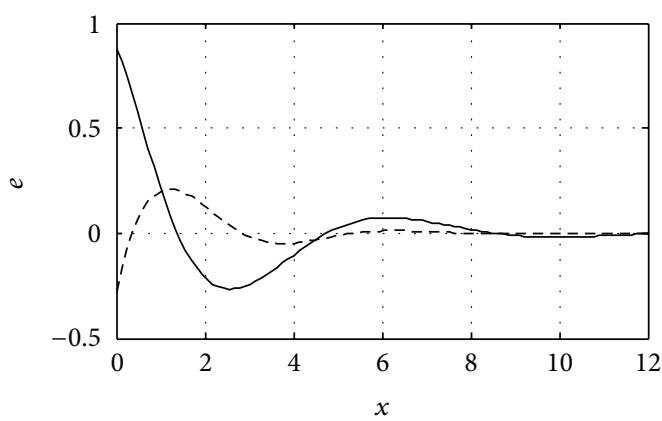

(d) The strain distribution (e)

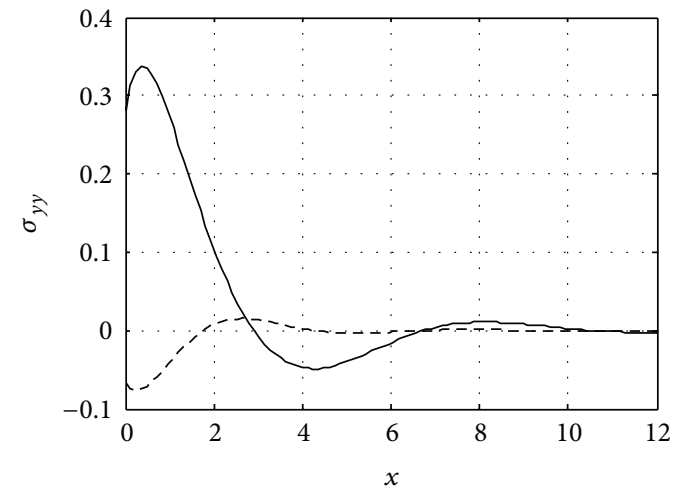

(f) The stress $\sigma_{y y}$ distribution

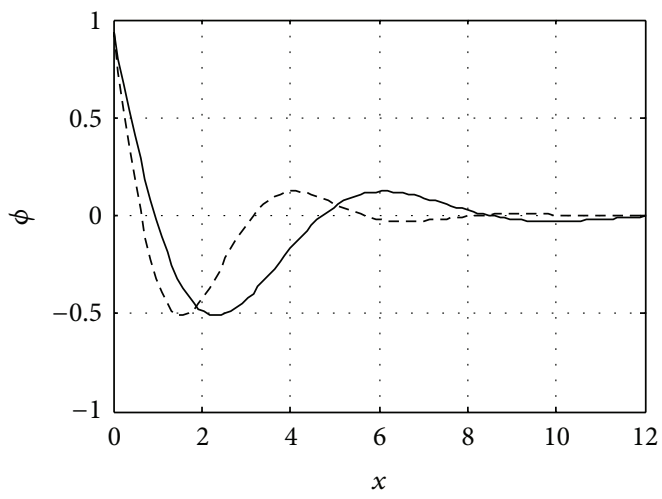

$\Omega=0.0$

$--\Omega=0.2$

(h) The conductive heat distribution

FIGURE 2: The thermal temperature $\theta$, the displacement $u, v$ the strain $e$ distribution, the stresses distribution $\sigma_{y y}, \sigma_{x x}$, and $\sigma_{x y}$ and the thermal temperature $\phi$ distribution with different values of rotation when $\beta=0.1$ and $t=0.1$. 
behaviour. The lines for $\Omega=0.0$ has the highest gradient when compared with that of $\Omega=0.2$. These trends obey elastic and thermoelastic properties of the solid. Figure 2(h) displays the conductive temperature in which we observe the significant difference in the conductive temperature that is noticed for the value of rotation. The conductive temperature begins from the positive values and then decreases to arrive the minimum amplitudes in two cases of $\Omega$, also move in the wave propagation, beyond it falls again to try to retain zero at infinity.

The third group Figures 3(a), 3(b), 3(c), 3(d), 3(e), 3(f), $3(\mathrm{~g})$, and $3(\mathrm{~h})$ show the comparison between the thermal temperature $\theta$ and $\phi$, displacement components ( $u$ and $v)$, strain distribution, and the stress $\left(\sigma_{x x}, \sigma_{y y}, \sigma_{x y}\right)$ distribution, the case of different two values of two temperature parameter. For the value of $y$, namely, $y=-1$ were substituted in performing the computation. Figure 3(a) exhibits the space variation of temperature distribution in which we observe the following: Significant difference in the thermodynamical temperature is noticed for different value of the nondimensional two-temperature parameter. It should be noted (Figure 3(a)). It is clear from the graph that $\theta$ has decreased to arrive the minimum value at the beginning in two cases $\beta=0.1$ and $\beta=0.2$ (two temperature). The value of temperature quantity converges to zero with increasing the distance $x$ and satisfies the boundary conditions at $x=0$. Also from this figure we can see when $\beta=0.1$ and $\beta=0.2$ move in the wave function.

In Figure 3(b) the horizontal displacement $u$, we see that the displacement component $u$ always starts from the negative value when $\beta=0.1$ and $\beta=0.2$ and terminates at the zero value begins with increase (then smooth increases) to reach its maximum magnitude. Beyond it $u$ falls again to try to retain zero at infinity, beyond reaching zero at the infinity (state of particles equilibrium). The displacements $u$ show the same behaviours at different values of $\beta$. In Figure 3(c), the vertical displacement $v$, we see that the displacement component $v$ always starts from the positive value and terminates at the zero value to reach the minimum value, beyond reaching zero at infinity with increases of $x$. Figure 3(d) displays a comparison of the strain in the context of two cases which show the same behaviours when $\beta=0.1$ and $\beta=0.2$. We can say that significant difference in the strain is noticed for different values of the non-dimensional two-temperature parameter. In addition, all lines begin to coincide when the horizontal distance $x$ increases to reach zero at infinity. The strain distribution is continuous, smooth, and moves in the wave function. These trends obey elastic and thermoelastic properties of the solid. The stress component, $\sigma_{x x}$, reach coincidence with zero value (Figure 3(e)) and reaches the maximum value in the beginning and smooth decreases then converges to zero with increasing the distance $x$. Figure 3(f) shows that the stress component $\sigma_{y y}$, increases in the start and arrive to maximum in the context of the two values of $\beta$. These trends obey elastic and thermoelastic properties of the solid under investigation. In Figure 3(g) the stress component $\sigma_{x y}$ satisfies the boundary condition and starts from zero. It sharp decreases in the start to arrive the minimum and then start smooth increases to maximum when $\beta=0.1$ but sharp increases in the start to arrive the maximum and then start smooth decreases to minimum when $\beta=0.2$. Figure $3(\mathrm{~h})$ displays the conductive temperature in which we observe the significant difference in the conductive temperature that is noticed for the value of the non-dimensional two temperature parameter $\beta$ where the case of $\beta=0.1$ and $\beta=0.2$ indicates the new case (two-temperature). The conductive temperature begins from the positive values and then decreases to arrive the minimum amplitudes in two cases of $\beta$, also move in the wave propagation when $\beta=0.1$ and $\beta=0.2$, beyond it falls again to try to retain zero at infinity.

The forth group Figures 4(a), 4(b), 4(c), 4(d), 4(e), 4(f), $4(\mathrm{~g})$, and $4(\mathrm{~h})$ show the comparison between the temperature $\theta$, the displacement components $u$ and the force stresses components $\sigma_{x x}$ and $\sigma_{x y}$, and the case of different two values of time, (namely, $t=0.1$ and $t=0.3$ ) under Lord-Şhulman (LS) theory. This group shows the effect of time on the results and we found that the curve when $t=0.1$ is greater than the curves when $t=0.2$ in all figures. These results obey physical reality for the behaviour of copper as a polycrystalline solid.

\section{Conclusions}

(1) The curves of the physical quantities with (CD) theory in most of figures are lower in comparison with those under (LS) theory, due to the relaxation times.

(2) Analytical solutions based upon normal mode analysis for thermoelastic problem in solids have been developed and utilized.

(3) The theory of two-temperature generalized thermoelasticity describes the behavior of the particles of the elastic body more real than the theory of onetemperature generalized thermoelasticity.

(4) In the context of the theory of two-temperature, the physical functions are continuous.

(5) The value of all the physical quantities converges to zero with an increase in distance $x$ and all functions are continuous.

(6) Deformation of a body depends on the nature of forced applied as well as the type of boundary conditions.

(7) It is clear from all the figures that all the distributions considered have nonzero value only in a bounded region of the half-space. Outside of this region, the values vanish identically and this means that the region has not felt thermal disturbance yet.

(8) From the temperature distributions, we have found a wave type heat propagation with finite speeds in the medium. The heat wave front moves forward with a finite speed in the medium with the passage of time which proves that the generalized thermoelasticity theory with two temperature heat transfer is very close to the behavior of the elastic materials. This is not the case for the CD theory where an infinite speeds of thermal propagation can be found and hence all the considered physical quantities have a nonzero (possibly very small) value for any point 


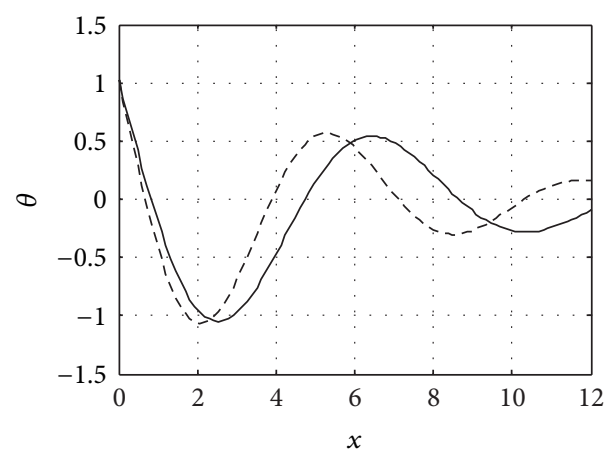

(a) The thermodynamical heat distribution

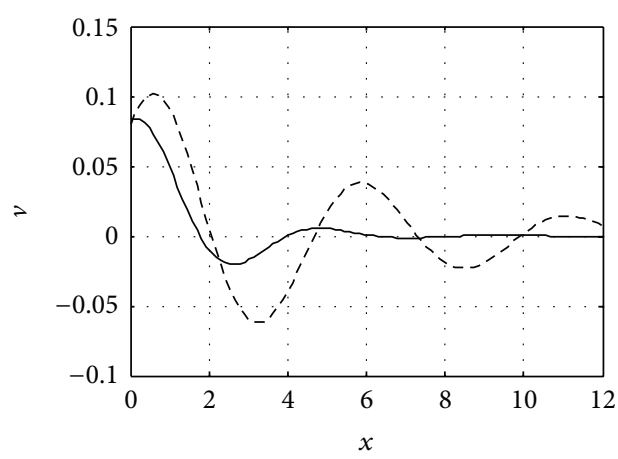

(c) The displacement distribution $(v)$

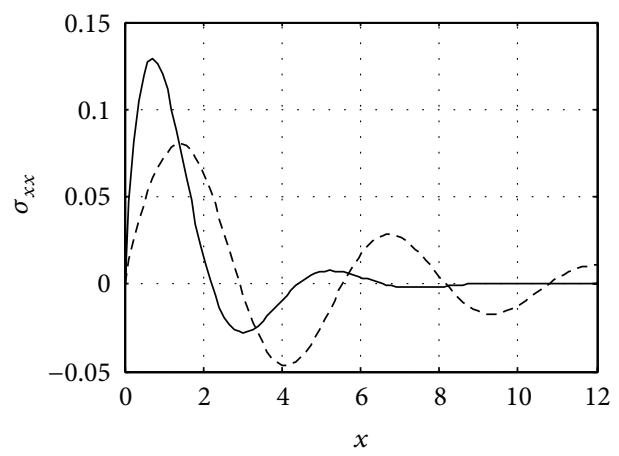

(e) The stress $\sigma_{x x}$ distribution

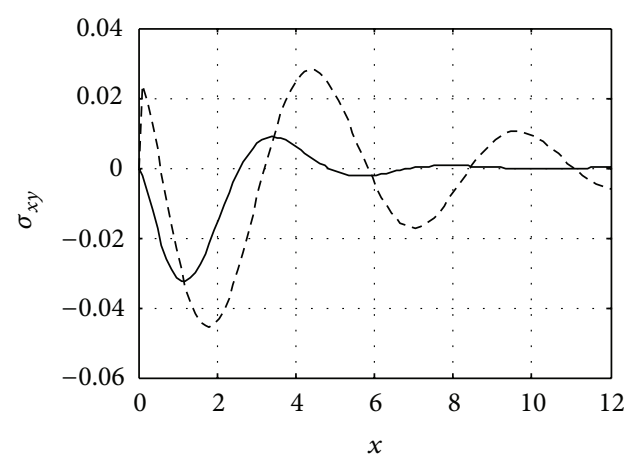

$-\beta=0.1$

$--\beta=0.2$

(g) The stress $\sigma_{x y}$ distribution

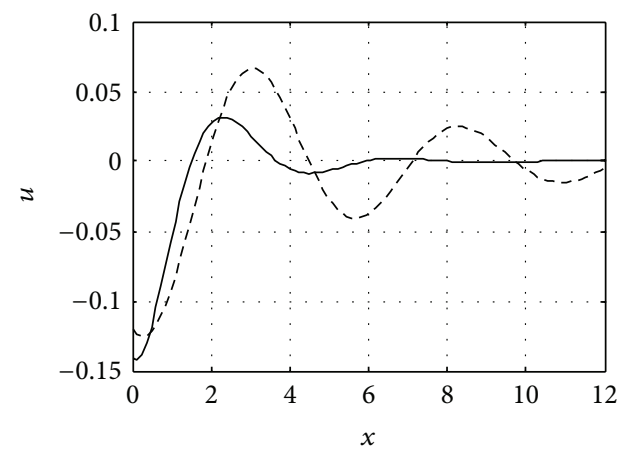

(b) The displacement distribution $(u)$

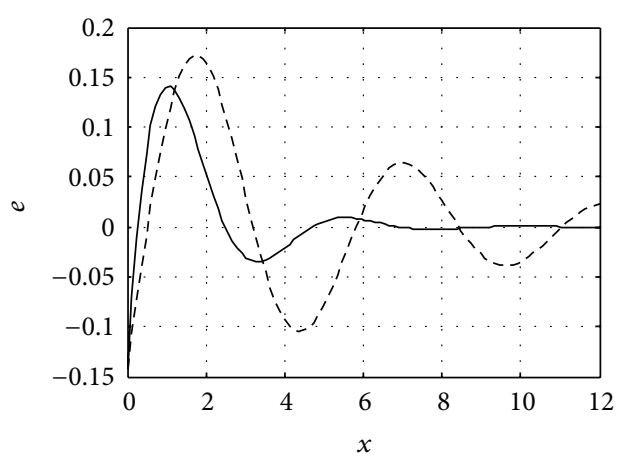

(d) The strain distribution (e)

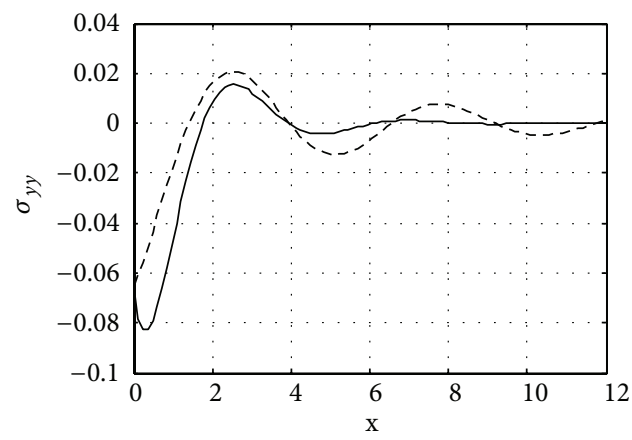

(f) The stress $\sigma_{y y}$ distribution

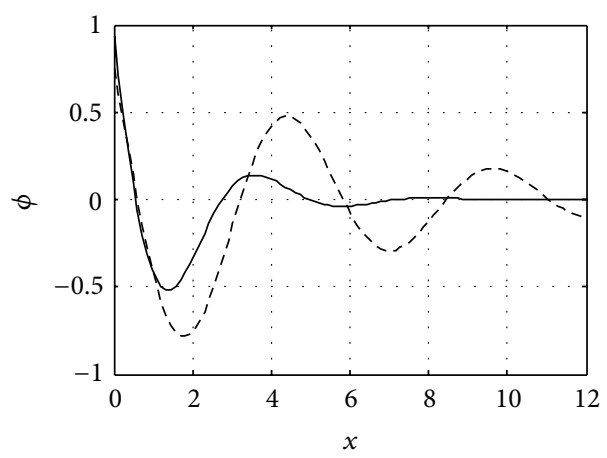

$-\beta=0.1$

- - $\beta=0.2$

(h) The conductive heat distribution

FIGURE 3: The thermal temperature $\theta$, the displacement $u, v$ the strain $e$ distribution, the stresses distribution $\sigma_{y y}, \sigma_{x x}$, and $\sigma_{x y}$ and the thermal temperature $\phi$ distribution with different values of two-temperature parameter at the constants $\Omega=0.2$ and $t=0.1$. 


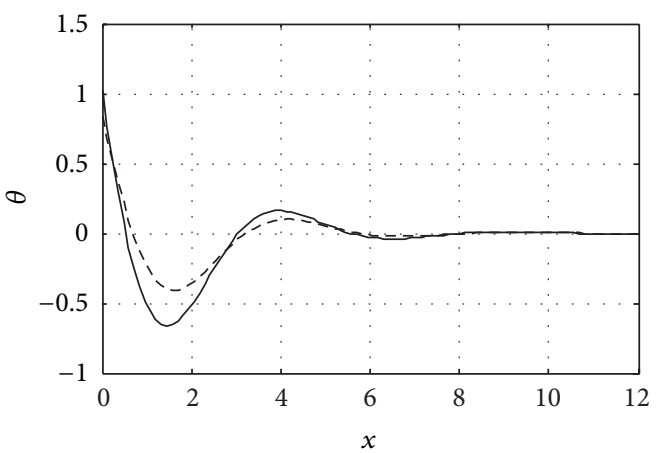

(a) The thermodynamical heat distribution

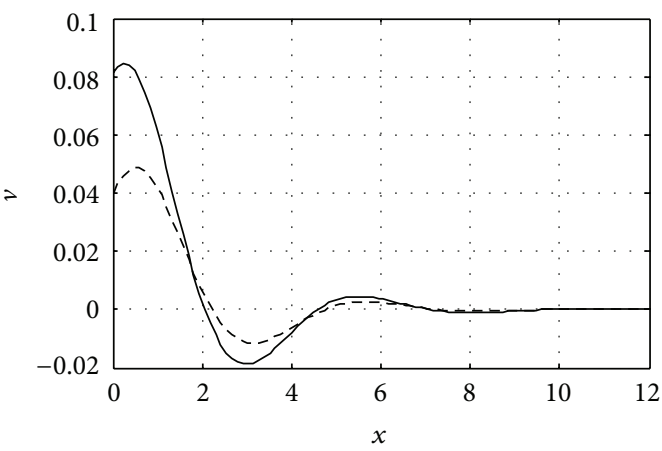

(c) The displacement distribution $(v)$

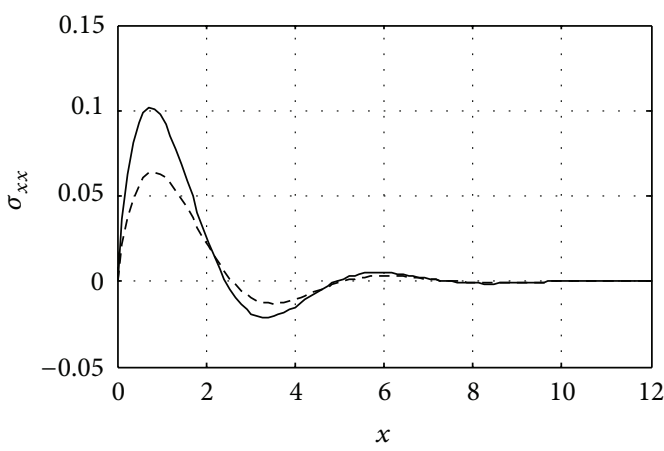

(e) The stress $\sigma_{x x}$ distribution

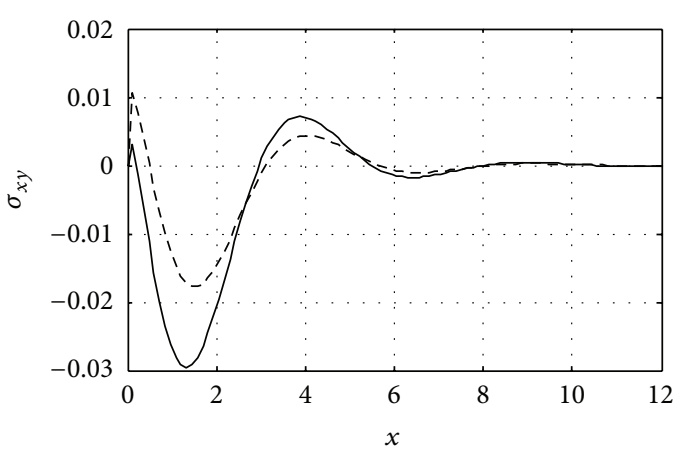

$-t=0.1$

(g) The stress $\sigma_{x y}$ distribution

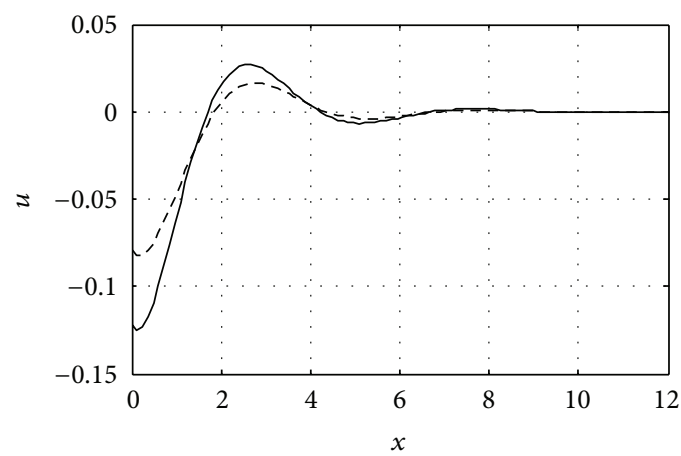

(b) The displacement distribution $(u)$

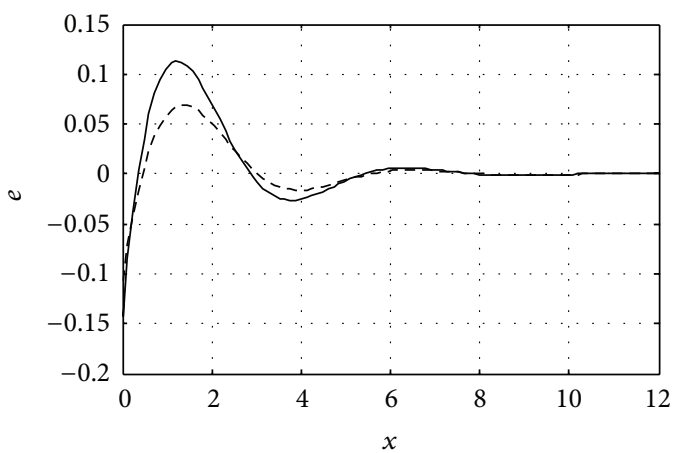

(d) The strain distribution $(e)$

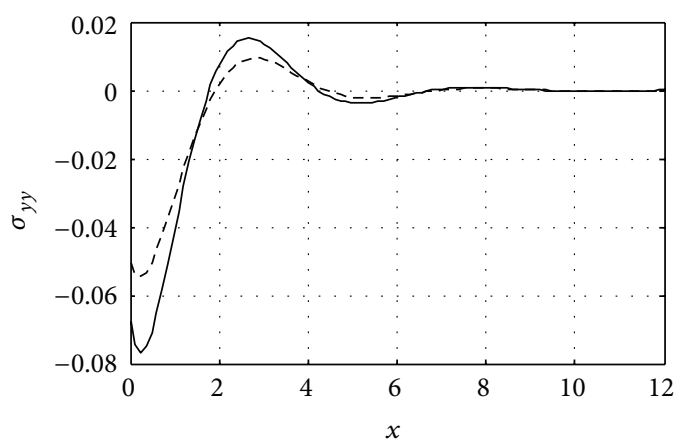

(f) The stress $\sigma_{y y}$ distribution

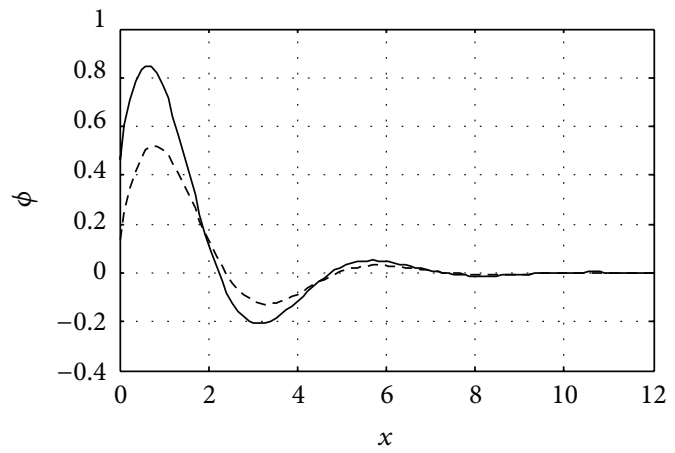

$-t=0.1$

$--t=0.3$

(h) The conductive heat distribution

FIGURE 4: The thermal temperature $\theta$, the displacement $u, v$ the strain $e$ distribution, the stresses distribution $\sigma_{y y}, \sigma_{x x}$, and $\sigma_{x y}$ and the thermal temperature $\phi$ distribution with different values of time at the constants $\Omega=0.2$ and $\beta=0.1$. 
in the medium. This indicates that the generalized Fourier's heat conduction mechanism is completely different from the classical Fourier's law.

\section{Nomenclature}

$\begin{array}{ll}\lambda, \mu: & \text { Counterparts of Lame's parameters } \\ p: & \text { Initial pressure } \\ \eta: & \text { Initial stress parameter } \\ a: & \text { Two temperature parameter } \\ \alpha_{t}: & \text { Coefficient of linear thermal expansion } \\ \theta=T-T_{0}: & \text { Thermodynamical temperature } \\ \phi=\phi_{0}-T: & \text { Conductive temperature } \\ T: & \text { Absolute temperature } \\ T_{0}: & \text { Temperature of the medium in its natural } \\ & \text { state assumed to be }\left|\left(T-T_{0}\right) / T_{0}\right|<1 \\ \sigma_{i j}: & \text { Components of the stress tensor } \\ u_{i}: & \text { Components of the displacement vector } \\ \rho: & \text { Density of the medium } \\ e_{i j}: & \text { Components of the strain tensor } \\ e: & \text { Cubical dilatation } \\ C_{E}: & \text { Specific heat at constant strain } \\ K: & \text { Thermal conductivity } \\ \tau_{0}: & \text { Thermal relaxation time } \\ \mu_{0}: & \text { Magnetic permeability } \\ \varepsilon_{0}: & \text { Electric permittivity } \\ F_{i}: & \text { Lorentz force } \\ \delta_{i j}: & \text { Kronecker delta function. } \\ & \end{array}$

\section{References}

[1] M. A. Biot, "Thermoelasticity and irreversible thermodynamics," Journal of Applied Physics, vol. 27, pp. 240-253, 1956.

[2] H. W. Lord and Y. Shulman, "A generalized dynamical theory of thermoelasticity," Journal of the Mechanics and Physics of Solids, vol. 15, no. 5, pp. 299-309, 1967.

[3] A. E. Green and K. A. Lindsay, "Thermoelasticity," Journal of Elasticity, vol. 2, no. 1, pp. 1-7, 1972.

[4] D. S. Chandrasekharaiah and K. S. Srinath, "Thermoelastic interactions without energy dissipation due to a point heat source," Journal of Elasticity, vol. 50, no. 2, pp. 97-108, 1998.

[5] D. S. Chandrasekharaiah and H. N. Murthy, "Temperature-ratedependent thermoelastic interactions due to a line heat source," Acta Mechanica, vol. 89, no. 1-4, pp. 1-12, 1991.

[6] P. Puri, "Plane waves in thermoelasticity and magneto-thermoelasticity," International Journal of Engineering Science, vol. 10, no. 5, pp. 467-477, 1972.

[7] A. Nayfeh and S. Nemat-Nasser, "Transient thermoelastic waves in a half-space with thermal relaxation," Zeitschrift für Angewandte Mathematik und Physik, vol. 23, no. 1, pp. 50-68, 1972.

[8] S. K. Roy Choudhuri and S. Mukhopdhyay, "Effect of rotation and relaxation on plane waves in generalized thermoviscoelasticity," International Journal of Mathematics and Mathematical Sciences, vol. 23, pp. 479-505, 2000.

[9] M. A. Ezzat and M. I. A. Othman, "Electromagneto-thermoelastic plane waves with two relaxation times in a medium of perfect conductivity," International Journal of Engineering Science, vol. 38, no. 1, pp. 107-120, 2000.

[10] M. Ezzat, M. I. A. Othman, and A. S. El-Karamany, "Electromagneto-thermoelastic plane waves with thermal relaxation in a medium of perfect conductivity," Journal of Thermal Stresses, vol. 24, no. 5, pp. 411-432, 2001.

[11] L. Y. Bahar and R. B. Hetnarski, "State space approach to thermoelasticity," in Proceedings of the 6th Canadian Congress of Applied Mechanics, pp. 17-18, University of British Columbia, Vancouver, Canada, 1977.

[12] L. Y. Bahar and R. B. Hetnarski, "Transfer matrix approach to thermoelasticity," in Proceedings of the 15th Midwest Mechanical Conference, pp. 161-163, University of Illinois at Chicago, Chicago, Ill, USA, 1977.

[13] L. Y. Bahar and R. Hetnarski, "State space approach to thermoelasticity," Journal of Thermal Stresses, vol. 1, pp. 135-145, 1978.

[14] H. H. Sherief, "State space formulation for generalized thermoelasticity with one relaxation time including heat sources," Journal of Thermal Stresses, vol. 16, no. 2, pp. 163-180, 1993.

[15] H. Sherief and M. Anwar, "Two-dimensional generalized thermoelasticity problem for an infinitely long cylinder," Journal of Thermal Stresses, vol. 17, no. 2, pp. 227-217, 1994.

[16] H. M. Youssef and A. A. El-Bary, "Mathematical model for thermal shock problem of a generalized thermoelastic layered composite material with variable thermal conductivity," Computational Methods in Science and Technology, vol. 12, no. 2, pp. 165-171, 2006.

[17] K. A. Elsibai and H. M. Youssef, "State-space approach to vibration of gold nano-beam induced by ramp type heating without energy dissipation in femtoseconds scale," Journal of Thermal Stresses, vol. 34, no. 3, pp. 244-263, 2011.

[18] P. J. Chen and M. E. Gurtin, "On a theory of heat conduction involving two temperatures," Zeitschrift für Angewandte Mathematik und Physik, vol. 19, no. 4, pp. 614-627, 1968.

[19] P. J. Chen and W. O. Williams, "A note on non-simple heat conduction," Zeitschrift für Angewandte Mathematik und Physik, vol. 19, no. 6, pp. 969-970, 1968.

[20] P. J. Chen, M. E. Gurtin, and W. O. Williams, "On the thermodynamics of non-simple elastic materials with two temperatures," Zeitschrift für Angewandte Mathematik und Physik, vol. 20, no. 1, pp. 107-112, 1969.

[21] J. K. Chen, J. E. Beraun, and C. L. Tham, "Ultrafast thermoelasticity for short-pulse laser heating," International Journal of Engineering Science, vol. 42, no. 8-9, pp. 793-807, 2004.

[22] T. Q. Quintanilla and C. L. Tien, "Heat transfer mechanism during short-pulse laser heating of metals," Journal of Heat Transfer, vol. 115, pp. 835-841, 1993.

[23] H. M. Youssef, "Theory of two-temperature-generalized thermoelasticity," IMA Journal of Applied Mathematics, vol. 71, no. 3, pp. 383-390, 2006.

[24] H. M. Youssef and E. A. Al-Lehaibi, "State-space approach of two-temperature generalized thermoelasticity of onedimensional problem," International Journal of Solids and Structures, vol. 44, no. 5, pp. 1550-1562, 2007.

[25] J. C. Misra, S. B. Kar, and S. C. Samanta, "Effects of mechanical and thermal relaxations on the stresses in a heated viscoelastic continuum with a cylindrical hole," Transactions of the Canadian Society for Mechanical Engineering, vol. 11, no. 3, pp. 151159, 1987.

[26] B. Singh, "Propagation of Rayleigh wave in a two-temperature generalized thermoelastic solid half-space," ISRN Geophysics, vol. 2013, Article ID 857937, 6 pages, 2013.

[27] B. Singh and K. Bala, "On Rayleigh wave in two-temperature generalized thermoelastic medium without energy dissipation," Applied Mathematics, vol. 4, no. 1, pp. 107-112, 2013. 
[28] S. K. R. Choudhuri and L. Debnath, "Magneto-thermoelastic plane waves in a rotating media," International Journal of Engineering Science, vol. 21, pp. 155-163, 1983.

[29] S. K. R. Choudhuri and L. Debnath, "Magneto-elastic plane waves in infinite rotating media," Journal of Applied Mechanics, vol. 50, pp. 283-288, 1983.

[30] M. I. A. Othman, "Effect of rotation on plane waves in generalized thermo-elasticity with two relaxation times," International Journal of Solids and Structures, vol. 41, no. 11-12, pp. 2939-2956, 2004.

[31] M. I. A. Othman, "Effect of rotation and relaxation time on a thermal shock problem for a half-space in generalized thermoviscoelasticity," Acta Mechanica, vol. 174, no. 3-4, pp. 129-143, 2005.

[32] M. I. A. Othman and B. Singh, "The effect of rotation on generalized micropolar thermoelasticity for a half-space under five theories," International Journal of Solids and Structures, vol. 44, no. 9, pp. 2748-2762, 2007.

[33] M. I. A. Othman and Y. Song, "Effect of rotation on plane waves of generalized electro-magneto-thermoviscoelasticity with two relaxation times," Applied Mathematical Modelling, vol. 32, no. 5, pp. 811-825, 2008.

[34] M. A. Ezzat and M. Z. Abd Elall, "Generalized magnetothermoelasticity with modified Ohm's law," Mechanics of Advanced Materials and Structures, vol. 17, no. 1, pp. 74-84, 2010.

[35] M. I. A. Othman and Kh. Lotfy, "On the plane waves of generalized thermo-microstretch elastic half-space under three theories," International Communications in Heat and Mass Transfer, vol. 37, no. 2, pp. 192-200, 2010.

[36] M. I. A. Othman, Kh. Lotfy, and R. M. Farouk, "Generalized thermo-microstretch elastic medium with temperature dependent properties for different theories," Engineering Analysis with Boundary Elements, vol. 34, no. 3, pp. 229-237, 2010.

[37] M. Othman and Kh. Lotfy, "The effect of magnetic field and rotation of the 2-D problem of a fiber-reinforced thermoelastic under three theories with influence of gravity," Mechanics of Materials, vol. 60, pp. 120-143, 2013.

[38] Kh. Lotfy and W. Hassan, "A mode-I crack problem for two-dimensional problem of a fiber-reinforced thermoelastic with normal mode analysis," International Journal of Physical Sciences, vol. 8, no. 22, pp. 1228-1245, 2013.

[39] Kh. Lotfy and M. Othman, "The effect of rotation on plane waves in generalized thermo-microstretch elastic solid with one relaxation time for a mode-I crack problem," Chinese Physics $B$, vol. 20, no. 7, Article ID 074601, 2011.

[40] Kh. Lotfy, "Mode-I crack in a two-dimensional fibre-reinforced generalized thermoelastic problem," Chinese Physics B, vol. 21, no. 1, Article ID 014209, 2012.

[41] N. Sarkar and A. Lahiri, "A three-dimensional thermoelastic problem for a half-space without energy dissipation," International Journal of Engineering Science, vol. 51, pp. 310-325, 2012. 


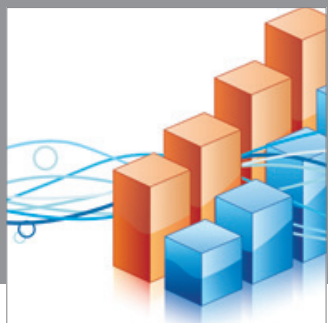

Advances in

Operations Research

mansans

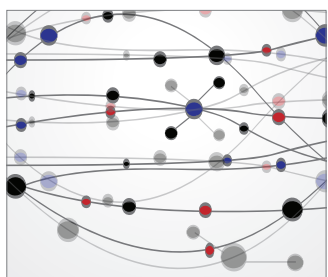

The Scientific World Journal
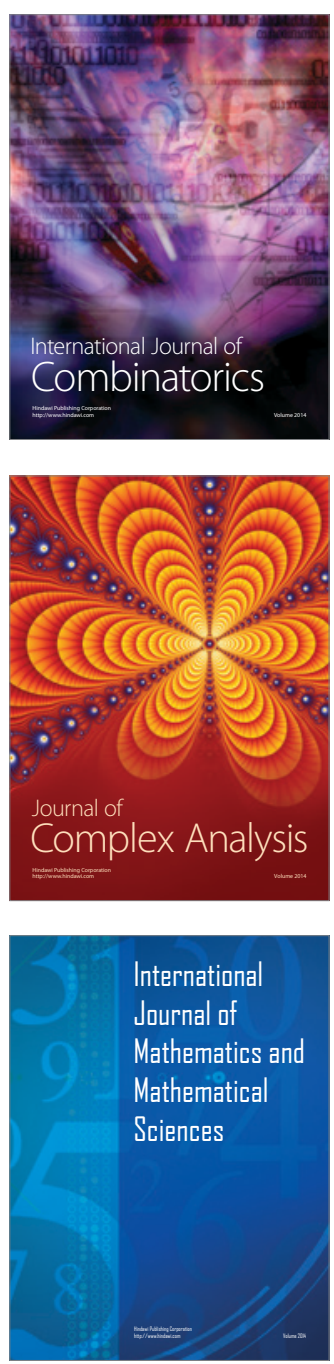
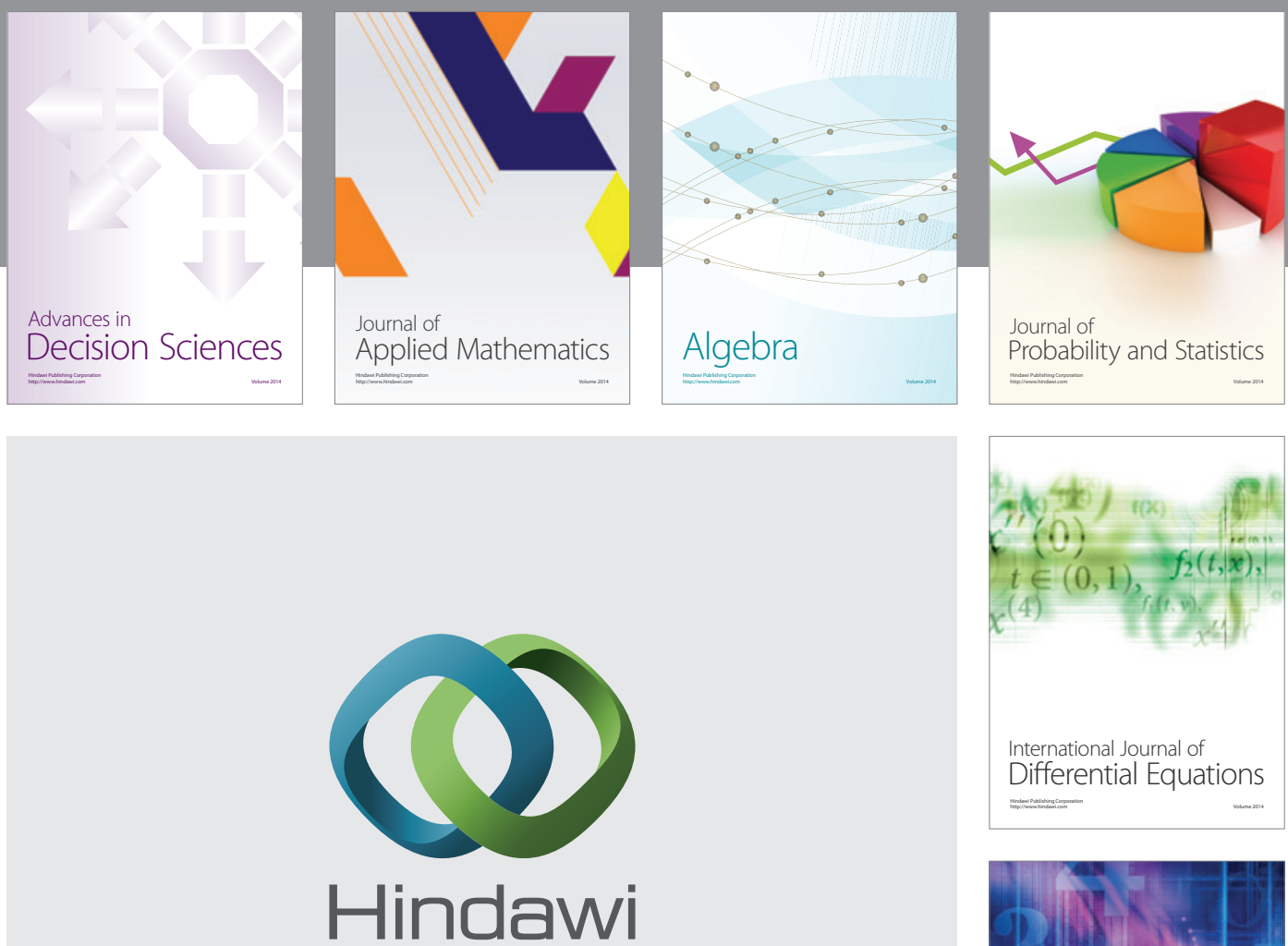

Submit your manuscripts at http://www.hindawi.com
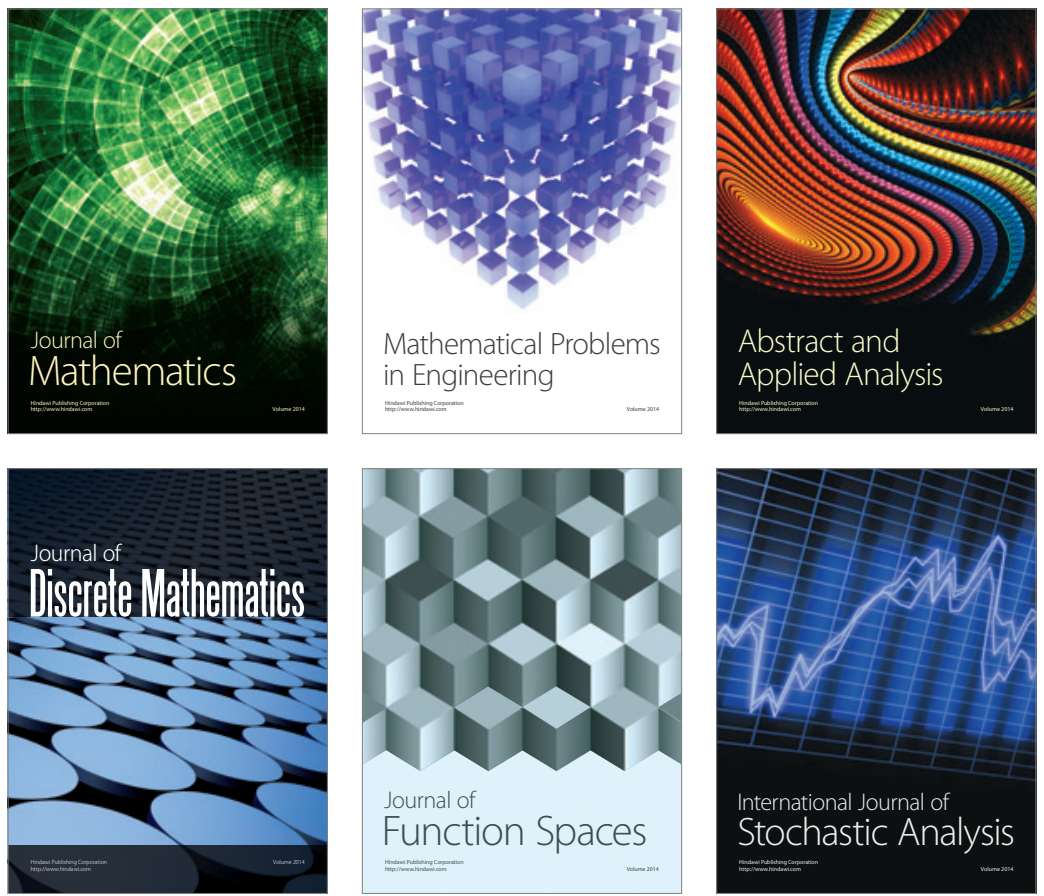

Journal of

Function Spaces

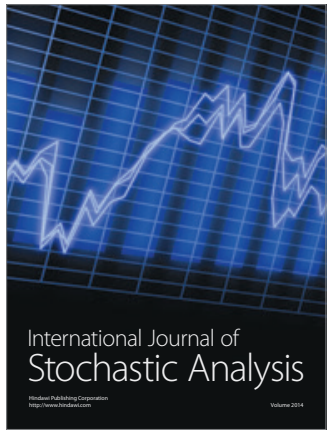

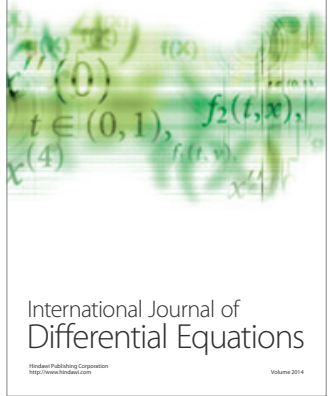
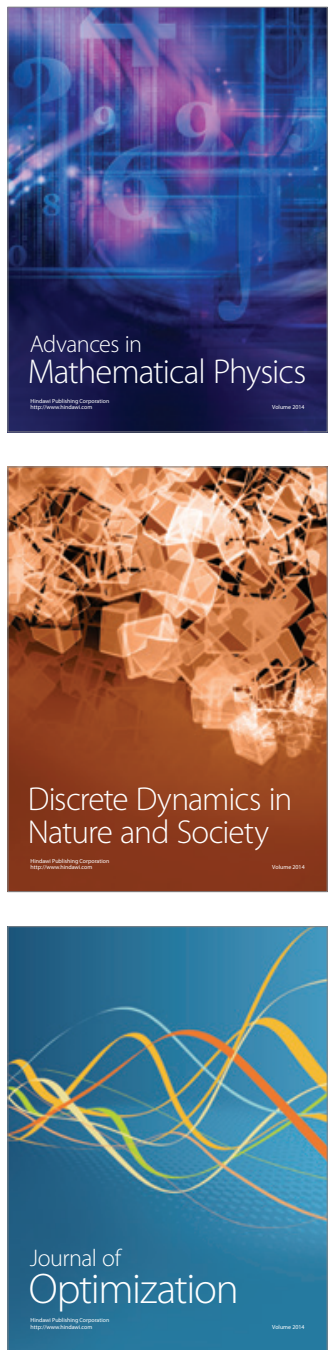